\title{
The introduction of automatic blood pressure monitoring to an accident \& emergency resuscitation room
}

\author{
S. CUSACK, C. MOULTON, and I. J. SWANN
}

Department of Accident and Emergency Medicine, Glasgow Royal Infirmary, 84 Castle Street, Glasgow

\section{SUMMARY}

Machines for automatic non-invasive blood pressure (BP) monitoring are increasingly available in British accident and emergency departments. Our department recently acquired two machines with this capability for use in the resuscitation room. This provided us with an opportunity to compare the speed and frequency of automatic BP recording with the previously used manual method. We found no significant difference in either the median time to the first recording of $\mathrm{BP}$ or in the median number of documented recordings in the first hour. However, the overall frequency of BP recording did show a statistically significant increase. We conclude that automation alone does not improve standard practice in this area greatly.

\section{INTRODUCTION}

The measurement of arterial BP forms an important part of the assessment of the critically ill patient (Marino, 1991). Automatic non-invasive BP recording is now widely used in A\&E department resuscitation rooms. Machines for this purpose are expensive. Their advantages appear to be in the freeing of hands to allow other important tasks to be undertaken and their ability to record BP accurately and consistently in noisy surroundings, thus providing frequent, regular measurements of an important vital sign.

Glasgow Royal Infirmary A\&E Department is a busy teaching hospital unit seeing over 75000 new patients annually. The unit has recently benefited from a

Correspondence: $\mathrm{Mr}$ S. Cusack, Senior Registrar, Department of Accident and Emergency Medicine, Glasgow' Royal Infirmary, 84 Castle Street, Glasgow G4 OSF. 
$£ 1.5$ million upgrading which involved both work on the building fabric and $\stackrel{\mathbb{D}}{0}$ re-equipping of the department. As part of this re-equipping, the unit obtained $\underset{\mathbb{D}}{3}$ two Datex Cardiocap II (TM) CG-2CS multiparameter physiological monitors for ? use in the resuscitation area (Datex Instrumentarium, Finland). Each machine cost over $£ 10000$ and is capable of monitoring and displaying ECG, $\mathrm{S}_{\mathrm{A}} \mathrm{O}_{2}$ and $\mathrm{ETCO}_{2} \overrightarrow{\mathrm{C}}$ in addition to both intra-arterial and non-invasive BP.

We undertook this study to assess what effect the introduction of this equipment had on the speed and frequency of BP recording in our resuscitation room.

\section{METHODS}

The A\&E resuscitation room was equipped with automatic BP monitors in April 1991. Prior to this, BP was measured by nurses using manual mercury sphygmomanometers. Before the introduction of the new machines, data were collected, $\omega_{0}$ without the knowledge of the nursing staff, on patients triaged to the resuscitation $\frac{\rho}{5}$ area. Particular note was made of the time elapsed from arrival in this area until the first recording of the $\mathrm{BP}$ and of the number of recordings made during the patient's first hour in the department.

Following the introduction of the new monitors, 4 months were allowed to pass $\overrightarrow{0}$ to ensure that all the nursing staff had become familiar with its use. At the end of this time the same data were collected again, once again without the nurses. knowledge. All such data were collected by the same three doctors (two registrass and one senior registrar). Statistical analysis of the results was made using the Mann-Whitney U test.

\section{RESULTS}

Information was collected on 31 patients prior to the introduction of the new equipment (manual group) and in 30 patients following its installation (automatic group). The times from arrival in the resuscitation area until the first recording of $\dot{\delta}$ the BP are shown in Fig. 1. Times are expressed to the nearest $5 \mathrm{~min}$. The number $₹$ of recordings per patient made during the first hour in the department is shown 9 in Fig. 2.

The median time to first recording of BP in the manual group was $7 \mathrm{~min}$ and in the automatic group it was $6 \mathrm{~min}$. Statistically, there was no significant difference $\mathcal{N}$ in these times. For both groups the mean time was over $8 \mathrm{~min}$.

The median number of BP recordings in the first hour in the manual group was three and in the automatic group it was also three. However, analysis of the spread of the readings showed that there was a significantly greater number of $\bullet$ recordings in the automatic group $(P=0.0389)$. 


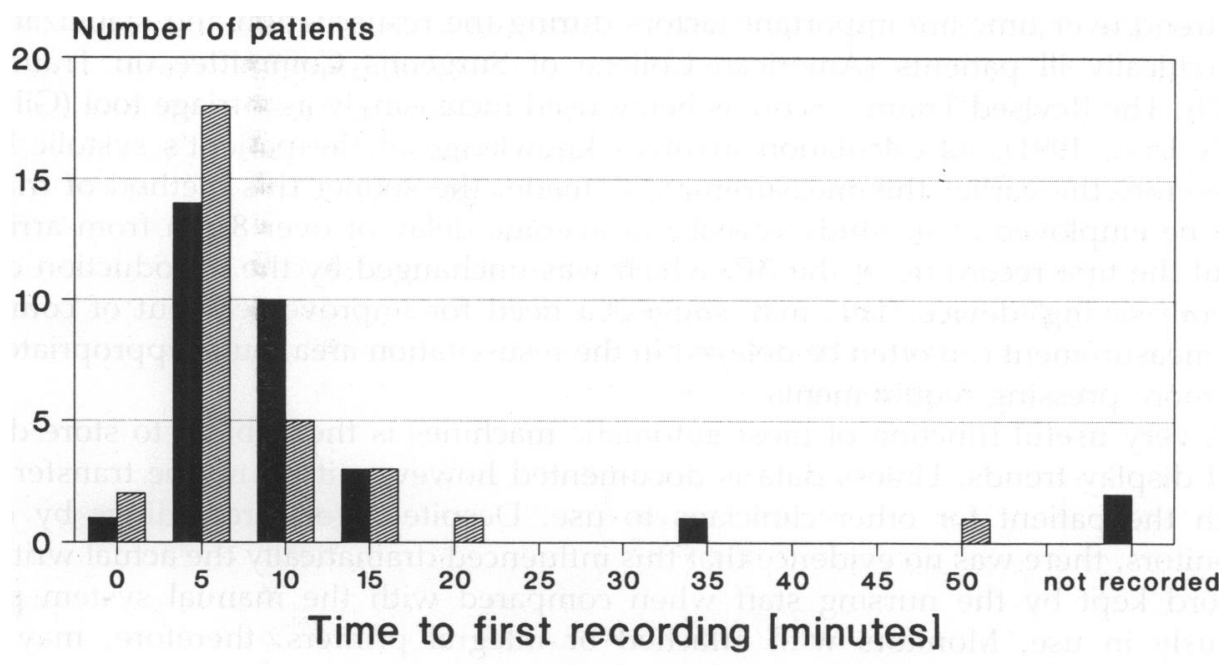

manual group [no=31] Wר: auto group [no=30]

Fig. 1. Time to first BP recording.

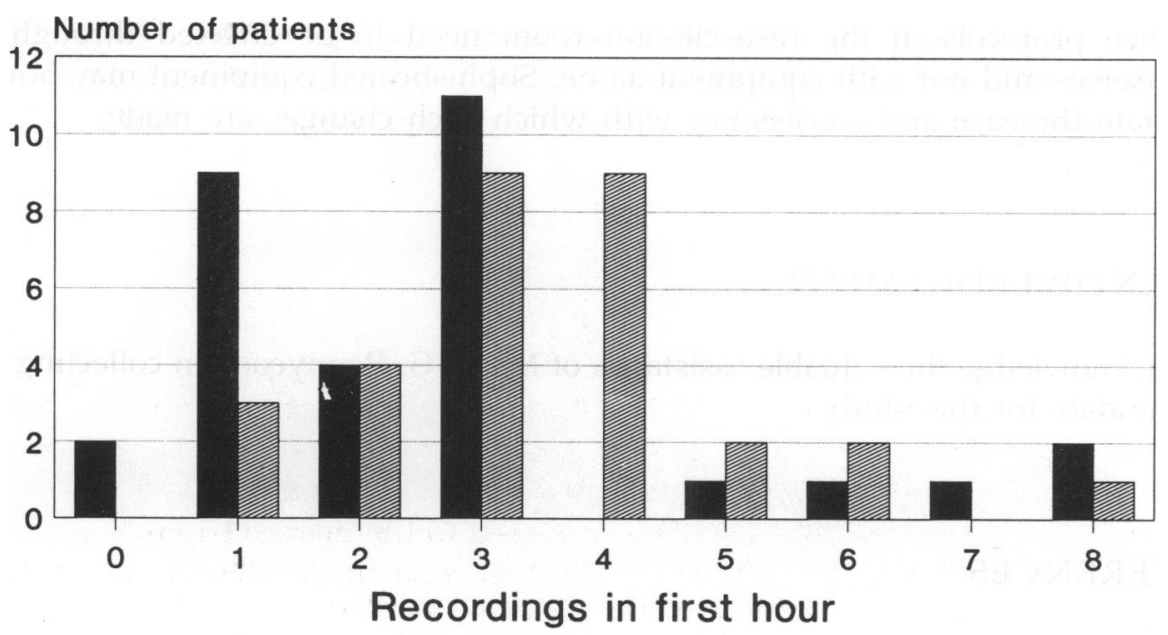

manual group [no=31] ऑon auto group [no=30]

Fig. 2. Number of BP recordings in first hour.

\section{DISCUSSION}

The accuracy of non-invasive BP measurement has been questioned (Cohn, 1967; Ryan et al., 1991). However, there is little doubt that the initial recording of BP and 
its trend over time are important factors during the resuscitation and stabilization $\frac{\mathbb{D}}{6}$ of critically ill patients (American College of Surgeons Committee on Trauma, 3 1989). The Revised Trauma Score is being used increasingly as a triage tool (Gilpin $\stackrel{\mathbb{Q}}{\circ}$ \& Nelson, 1991); its calculation involves knowledge of the patient's systolic BP. $\overrightarrow{\vec{s}}$ Therefore the earlier this measurement is made, the sooner this method of triage $\frac{?}{0}$ can be employed. This study reveals an average delay of over $8 \mathrm{~min}$ from arrivalo until the first recording of the $\mathrm{BP}$, which was unchanged by the introduction of a $\frac{\overline{\bar{c}}}{\bar{\sigma}}$ 'labour-saving' device. This may suggest a need for improvement but of course, $\stackrel{\unrhd}{\varrho}$ BP measurement can often be delayed in the resuscitation area, quite appropriately, by more pressing requirements.

A very useful function of most automatic machines is their ability to store data $\overrightarrow{\vec{H}}$ and display trends. Unless data is documented however, it cannot be transferred with the patient for other clinicians to use. Despite regular recordings by our $\frac{\Phi}{3}$. monitors, there was no evidence that this influenced dramatically the actual written record kept by the nursing staff when compared with the manual system pre- $-\circ$ viously in use. Monitors with attached or integral printers, therefore, may be $\omega_{0}$ of further value. Experienced nursing staff, however, may prefer to determine음 the frequency of recording vital signs for themselves. It is also quite probable that $\vec{z}$ the number of measurements made is influenced by requests for this information from medical staff.

In conclusion, this study suggests that the introduction of automated monitoring $\overrightarrow{0}$ equipment alone does not influence standard practice greatly. Any changes practice protocols in the resuscitation room need to be effected through staff themselves and not with equipment alone. Sophisticated equipment may howev facilitate the ease and consistency with which such changes are made.

\section{ACKNOWLEDGEMENT}

We acknowledge the valuable assistance of Mr A. G. Pennycook in collecting some of the data for this study.

\section{REFERENCES}

American College of Surgeons Committee on Trauma (1989) Advanced Trauma Life Support Student Manual, pp. 246. American College of Surgeons, Chicago.

Cohn J. N. (1967) Blood pressure measurement in shock. Mechanism of inaccuracy in auscultatory and palpatory methods. Journal of the American Medical Association 199, 972-976.

Gilpin D. A. \& Nelson P. G. (1991) Revised trauma score: a triage tool in the accident and emergency department. Injury 22, 35-37.

Marino P. L. (1991) The ICU Book, pp. 89-100. Lea and Febiger, Philadelphia.

Ryan B. P., Redmond A. D. \& Edwards J. D. (1991) When to stop resuscitation - the significance of cuff blood pressure. Archives of Energency Medicine 8, 177-181. 